\title{
Origin of the in-orbit instrumental background of the Hard X-ray Imager onboard Hitomi
}

\author{
Kouichi Hagino $\odot$, ${ }^{\mathrm{a}, *}$ Hirokazu Odaka, ${ }^{\mathrm{b}, \mathrm{c}, \mathrm{d}}$ Goro Sato, ${ }^{\mathrm{e}}$ Tamotsu Sato, \\ Hiromasa Suzuki $\odot,{ }^{\text {b }}$ Tsunefumi Mizuno $\odot,{ }^{\text {g,h }}$ Madoka Kawaharada, ${ }^{i}$ \\ Masanori Ohno, ${ }^{\text {g,j,k }}$ Kazuhiro Nakazawa, ${ }^{1}$ Shogo B. Kobayashi, ${ }^{m}$ \\ Hiroaki Murakami, ${ }^{\text {b }}$ Katsuma Miyake, ${ }^{\text {b }}$ Makoto Asai, ${ }^{\text {n }}$ Tatsumi Koi, ${ }^{\circ}$ \\ Greg Madejski, ${ }^{\text {n,p }}$ Shinya Saito, ${ }^{\mathrm{q}}$ Dennis H. Wright, ${ }^{\mathrm{n}}$ Teruaki Enoto ${ }^{\mathrm{r}}{ }^{\mathrm{r}}$ \\ Yasushi Fukazawa, ${ }^{\text {g }}$ Katsuhiro Hayashi, ${ }^{\text {J Jun Kataoka, }}$, \\ Junichiro Katsuta, ${ }^{\mathrm{g}}$ Motohide Kokubun, ${ }^{\mathrm{f}}$ Philippe Laurent $\odot$,, ,u \\ François Lebrun, ${ }^{\text {t,u }}$ Olivier Limousin, ", Daniel Maier $\odot,{ }^{\mathrm{u}}$ \\ Kazuo Makishima, ${ }^{\text {b,d,v }}$ Kunishiro Mori, ${ }^{\text {f }}$ Takeshi Nakamori, ${ }^{\text {w }}$ \\ Toshio Nakano, ${ }^{v}$ Hirofumi Noda,, ${ }^{x}$ Masayuki Ohta, ${ }^{f}$ Rie Sato, \\ Hiroyasu Tajima ${ }^{\mathrm{z}}{ }^{\mathrm{z}}$ Hiromitsu Takahashi,g Tadayuki Takahashi, ${ }^{\mathrm{b}, \mathrm{d}}$ \\ Shin'ichiro Takeda, ${ }^{d}$ Takaaki Tanaka, ${ }^{\text {a }}$ Yukikatsu Terada $\odot,{ }^{\text {ab }}$ \\ Hideki Uchiyama, ${ }^{\text {ac }}$ Yasunobu Uchiyama, ${ }^{\text {,ad }}$ Shin Watanabe, ${ }^{\text {d,f }}$

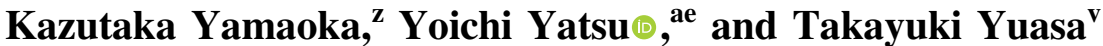 \\ ${ }^{a}$ Tokyo University of Science, Department of Physics, Noda, Chiba, Japan \\ ${ }^{\mathrm{b}}$ The University of Tokyo, Department of Physics, Tokyo, Japan
}

${ }^{c}$ The University of Tokyo, School of Science, Research Center for the Early Universe, Tokyo, Japan

${ }^{\mathrm{d}}$ Kavli Institute for the Physics and Mathematics of the Universe (WPI), The University of Tokyo Institutes for Advanced Study, The University of Tokyo, Kashiwa, Chiba, Japan 'Japan Aerospace Exploration Agency, Tokyo, Japan

${ }^{\mathrm{f} J a p a n}$ Aerospace Exploration Agency, Institute of Space and Astronautical Science, Sagamihara, Kanagawa, Japan

${ }^{\mathrm{g}}$ Hiroshima University, School of Science, Higashi-Hiroshima, Japan

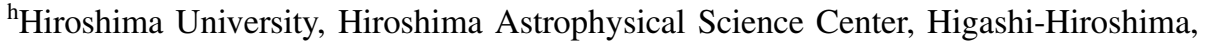
Hiroshima, Japan

${ }^{\mathrm{i} J a p a n}$ Aerospace Exploration Agency, Space Technology Directorate I, Tsukuba, Ibaraki, Japan ${ }^{\mathrm{j} E o ̈ t v o ̈ s ~ U n i v e r s i t y, ~ I n s t i t u t e ~ o f ~ P h y s i c s, ~ B u d a p e s t, ~ H u n g a r y ~}$

${ }^{k}$ MTA-Eötvös University Lendület Hot Universe and Astrophysics Research Group, Pázmány Budapest, Hungary

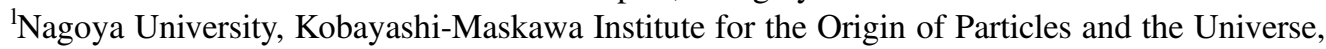
Nagoya, Japan

${ }^{\mathrm{m}}$ Tokyo University of Science, Department of Physics, Tokyo, Japan

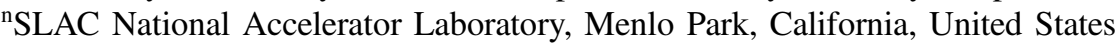

${ }^{\circ}$ Chubu University, College of Engineering, Kasugai, Aichi, Japan

${ }^{\mathrm{p}}$ Stanford University, Kavli Institute for Particle Astrophysics and Cosmology, Stanford, California, United States

${ }^{\mathrm{q}}$ Rikkyo University, Department of Physics, Tokyo, Japan

${ }^{r}$ RIKEN Cluster for Pioneering Research, Extreme Natural Phenomena RIKEN Hakubi Research Team, Wako, Saitama, Japan

${ }^{\mathrm{s}}$ Waseda University, Research Institute for Science and Engineering, Shinjuku, Tokyo, Japan

tUniversité de Paris, CNRS, Astroparticule et Cosmologie, Paris, France

"Université de Paris, CEA/DRF/IRFU/DAP_AIM, CEA/CNRS/Université Paris-Saclay, Gif-sur-Yvette, France

${ }^{v}$ High Energy Astrophysics Laboratory, Nishina Center, RIKEN, Saitama, Japan

${ }^{w}$ Yamagata University, Faculty of Science, Yamagata, Yamagata, Japan

${ }^{x}$ Osaka University, Department of Earth and Space Science, Toyonaka, Osaka, Japan

${ }^{y}$ Osaka University, Project Research Center for Fundamental Sciences, Toyonaka, Osaka, Japan 


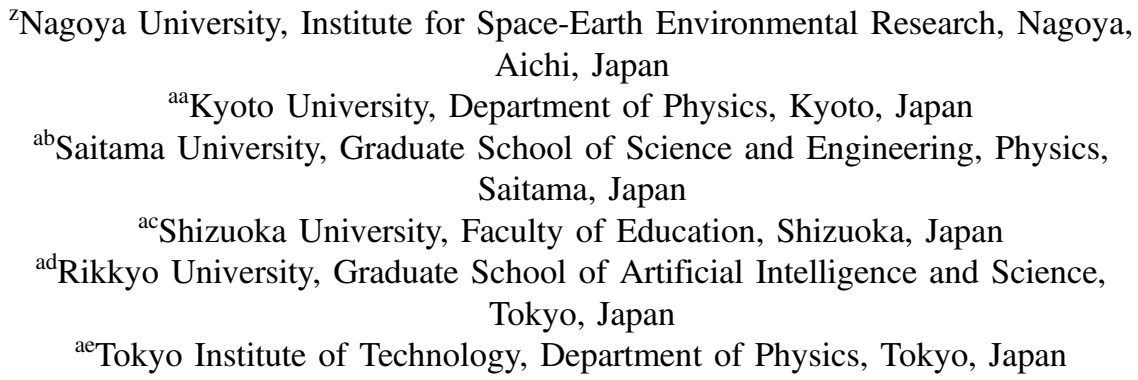

\begin{abstract}
Understanding and reducing in-orbit instrumental backgrounds are essential to achieving high sensitivity in hard x-ray astronomical observations. The observational data of the Hard X-ray Imager (HXI) onboard the Hitomi satellite provide useful information on the background components due to its multilayer configuration with different atomic numbers: the HXI consists of a stack of four layers of $\mathrm{Si}(Z=14)$ detectors and one layer of cadmium telluride (CdTe) $(Z=48,52)$ detector surrounded by well-type $\mathrm{Bi}_{4} \mathrm{Ge}_{3} \mathrm{O}_{12}$ active shields. Based on the observational data, the backgrounds of the top Si layer, the three underlying Si layers, and the CdTe layer are inferred to be dominated by different components, namely, low-energy electrons, albedo neutrons, and proton-induced radioactivation, respectively. Monte Carlo simulations of the in-orbit background of the HXI reproduce the observed background spectrum of each layer well, thereby quantitatively verifying the above hypothesis. In addition, we suggest the inclusion of an electron shield to reduce the background. () The Authors. Published by SPIE under a Creative Commons Attribution 4.0 Unported License. Distribution or reproduction of this work in whole or in part requires full attribution of the original publication, including its DOI. [DOI: 10.1117/1.JATIS.6.4.046003]
\end{abstract}

Keywords: Hitomi; ASTRO-H; hard x-ray imager; hard x-rays; background; simulation.

Paper 20057 received May 25, 2020; accepted for publication Nov. 19, 2020; published online Dec. 3, 2020.

\title{
1 Introduction
}

The hard x-ray imager (HXI) was one of the four observational instruments onboard the Hitomi satellite, which was launched on February 17, 2016. ${ }^{1,2}$ Combined with the hard x-ray telescopes, ${ }^{3}$ the HXI was designed to perform imaging spectroscopy in the 5- to $80-\mathrm{keV}$ band. Although the Hitomi satellite was lost on March 25, 2016, the HXI was operational for 13 days in orbit, providing useful insights on planning and designing future hard $\mathrm{x}$-ray missions.

As shown in Fig. 1, the HXI consists of a stack of four layers of silicon $(\mathrm{Si} ; Z=14)$ and one layer of cadmium telluride (CdTe; $Z=48,52$ ) semiconductor detectors surrounded by well-type BGO $\left(\mathrm{Bi}_{4} \mathrm{Ge}_{3} \mathrm{O}_{12}\right)$ active shields. ${ }^{5}$ The $\mathrm{BGO}$ active shields have a typical thickness of $\sim 3 \mathrm{~cm}$, and their design is similar to that of the hard $\mathrm{x}$-ray detector (HXD) onboard Suzaku, ${ }^{6,7}$ which efficiently reduced external backgrounds by the anticoincidence technique. The main imager is composed of a 0.5-mm-thick double-sided Si strip detector (DSSD) and a 0.75-mm-thick CdTe double-sided detector (CdTe-DSD), with an imaging area of $32 \times 32 \mathrm{~cm}^{2}$. Four layers of DSSDs achieve a detection efficiency of $>50 \%$ for $\mathrm{x}$-rays below $30 \mathrm{keV}$. Combined with the CdTe-DSD, a detection efficiency of $>70 \%$ is realized for $\mathrm{x}$-rays up to $80 \mathrm{keV}$. The $\mathrm{Si}$ detectors detect lowerenergy $\mathrm{x}$-rays with less radioactivation background than high- $Z$ detectors, while CdTe detectors detect higher-energy x-rays that cannot be absorbed by $\mathrm{Si}$. This stacked configuration of the main imager is effective in optimizing the in-orbit instrumental background and in achieving high sensitivity in the hard $x$-ray band.

The multilayer configuration of low- $Z$ ( $\mathrm{Si}$ ) and high- $Z$ (CdTe) sensors is very useful, not only in achieving high sensitivity, but also in decomposing the background components since interactions between the detector materials and cosmic-ray particles/photons have different

*Address all correspondence to Kouichi Hagino, hagino@rs.tus.ac.jp 


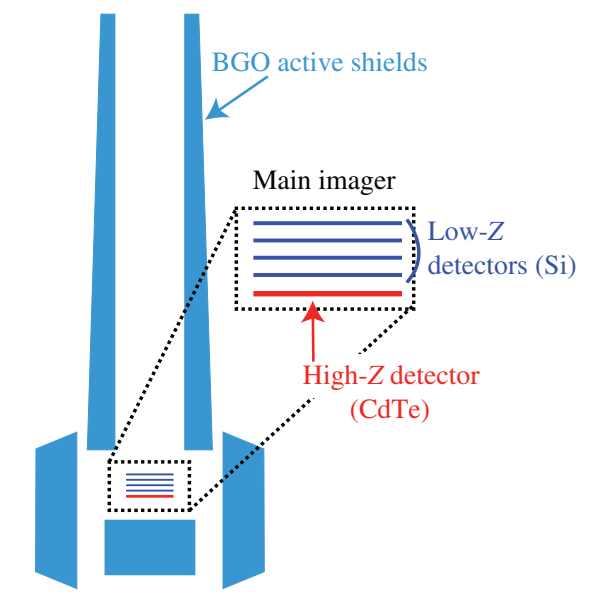

Fig. 1 Schematic structure of the HXI. This figure is adapted from Odaka et al. ${ }^{4}$

$Z$-dependences. For example, the high- $Z$ materials are more sensitive to $X / \gamma$-ray photons than the low- $Z$ materials because the photoabsorption crosssection is proportional to $Z^{4}$. On the other hand, high- $Z$ materials are less affected by neutrons because the recoil energy of nucleus via elastic scattering with neutrons is roughly proportional to the inverse of the atomic mass number $A$. Therefore, the multilayer configuration of the HXI would provide us with fruitful knowledge on the origins of the in-orbit instrumental backgrounds, which is essential to achieve high sensitivity in hard $\mathrm{x}$-ray astronomical observations.

In this paper, we describe our investigation of the origins of the HXI backgrounds using both observational data analysis and full Monte Carlo simulations. Section 2 describes the observational properties of the in-orbit instrumental backgrounds of the HXI. In Sec. 3, we describe the Monte Carlo simulations of the HXI and compare their results with the observational data. In Sec. 4, based on the results obtained from the simulations, we provide suggestions for future instruments in terms of reducing the in-orbit backgrounds, and finally, we provide conclusions in Sec. 5.

\section{Observational Properties of the HXI Background}

The observed background spectrum of each layer of the HXI and its dependence on the satellite position (latitude/longitude) are shown in Figs. 2 and 3. Since the background spectra of layers 1 to 3 (Fig. 2) are similar to each other, hereafter, we separate the detectors of the HXI into three groups: the top layer of the DSSD (layer 0), the remaining three layers of the DSSD (layer 1 to 3 ), and the CdTe-DSD layer (layer 4). In this section, we describe the observed properties of the backgrounds of each group and their origins as suggested by the observations.

\subsection{Electron Background in the Top-Layer DSSD}

The background spectrum of the top-layer DSSD (layer 0; red circle in Fig. 2) was a hard power law extending up to high energies of $\sim 100 \mathrm{keV}$. In spite of its very large energy deposit $(\sim 100 \mathrm{keV})$, such a component was not seen in the other layers, indicating that the origin of this background component has a low penetrating power, while having energy of more than $\sim 100 \mathrm{keV}$. This indicates that low-energy charged particles dominate the observed background.

In addition to the spectral properties, the variation of the count rate of this component with latitude and longitude of the satellite [Fig. 3(a)] matches well with the distribution of the geomagnetically-trapped electrons measured by the Instrument for Detecting Particles (IDP) onboard the Detection of Electro-Magnetic Emissions Transmitted from Earthquake Regions (DEMETER) satellite. ${ }^{8}$ This distribution is clearly different from that of the geomagnetically trapped protons, especially near the latitudes and longitudes corresponding to North America (latitude of $\gtrsim 20^{\circ}$ and longitude of $\lesssim-100^{\circ}$ ). Therefore, the background of the top-layer DSSD is considered to originate from the geomagnetically trapped low-energy electrons. 


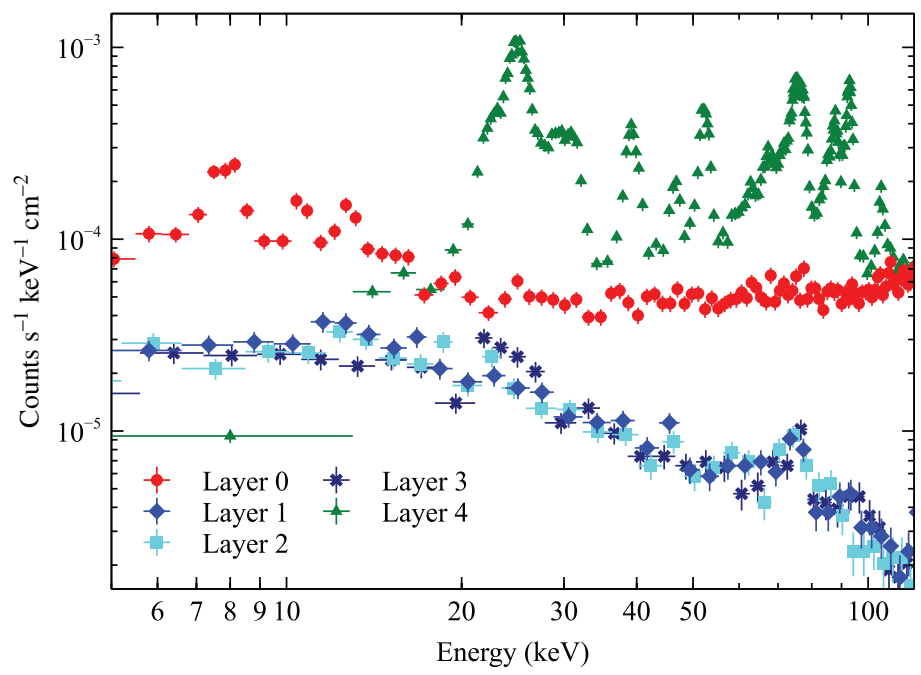

Fig. 2 Observed spectra of the instrumental background of the HXI, obtained from the Earth occultation data. It is worth noting that the vertical axis is per the area of the detector (not the mirror).

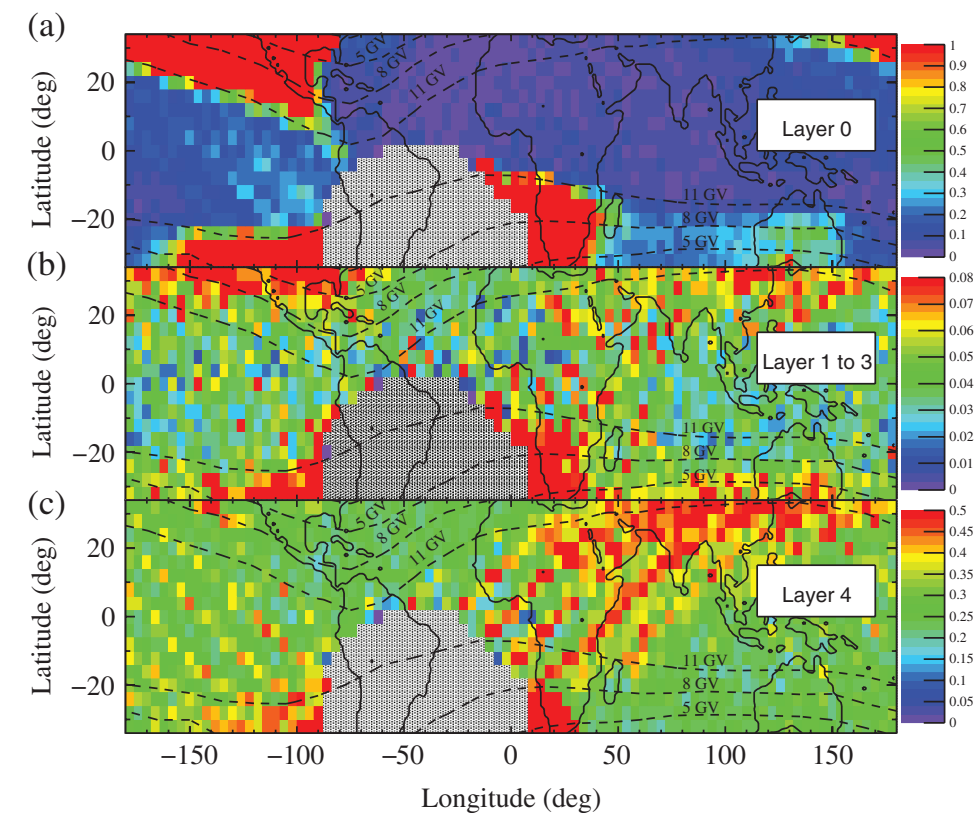

Fig. 3 Position dependence of the count rate (counts $\mathrm{s}^{-1}$ ) of the (a) top-layer DSSD (layer 0); (b) middle-layer DSSDs (layer 1 to 3); and (c) CdTe-DSD (layer 4) of the HXI during Earth occultation and blank sky observations. The black dashed lines indicate the geomagnetic cut-off rigidity. The gray region indicates the SAA where no data is downloaded from the satellite.

\subsection{Neutron Background in the Middle-Layer DSSDs}

The most remarkable property of the background spectra of the middle-layer DSSDs (layer 1 to 3 ) is their similarity to each other. Such a property would be exhibited if the incident particle has a high penetrating power. Moreover, this component could not originate from the high-energy charged particles since the charged particles would be rejected because of anticoincidence with the BGO active shields or with the other layers in onboard/on-ground data screening.

The similarity of the configuration of the detector ( $\mathrm{Si}$ detector surrounded by well-type BGO active shields) and that present in Suzaku/HXD ${ }^{6,7}$ suggests that the background component of these detectors should also be similar. In addition, after scaling the HXD and HXI detectors with their corresponding thicknesses $(0.2$ and $0.05 \mathrm{~cm}$, respectively), we find that the background 
of the $\mathrm{HXD} \mathrm{Si} \mathrm{detector} \mathrm{at} 10$ to $20 \mathrm{keV}\left(\sim 2 \times 10^{-4}\right.$ counts s $\mathrm{keV}^{-1} \mathrm{~cm}^{-2} / 0.2 \mathrm{~cm} \simeq$ $1 \times 10^{-3}$ counts s$\left.{ }^{-1} \mathrm{keV}^{-1} \mathrm{~cm}^{-3}\right)$ is consistent with that of the middle-layer DSSDs in HXI $\left(\sim 3 \times 10^{-5}\right.$ counts $\mathrm{s}^{-1} \mathrm{keV}^{-1} \mathrm{~cm}^{-2} / 0.05 \mathrm{~cm} \simeq 0.6 \times 10^{-3}$ counts s$\left.{ }^{-1} \mathrm{keV}^{-1} \mathrm{~cm}^{-3}\right)$ within a factor of two. In the case of the HXD Si detector, the background is thought to be dominated by albedo neutrons. ${ }^{9,10}$ One of the supporting evidences is that it shows an anticorrelation with the cut-off rigidity. In other words, the background of the HXD Si detector is higher at the locations with lower cut-off rigidity. Since the cut-off rigidity is a parameter describing the geomagnetic shielding from the cosmic-ray particles, the lower cut-off rigidity means that there are a lot of cosmic-ray particles and their secondary particles such as albedo neutrons. As shown in Fig. 3(b), the middle-layer DSSDs in HXI also have such a property. Therefore, similar to the HXD Si detector, the background of the middle-layer DSSDs in HXI should also be dominated by albedo neutrons.

\subsection{Proton-Induced Radioactivation Background in the CdTe-DSD}

Unlike the featureless spectrum of DSSDs, the background spectrum of CdTe-DSD (layer 4; green triangle in Fig. 2) is composed of many emission lines. Most of these lines originate from the radioactive isotopes arising from the activation of $\mathrm{Cd}$ and $\mathrm{Te}$, induced by geomagnetically trapped protons in the South Atlantic Anomaly (SAA). Although this effect is inevitable, it is mitigated by the thick BGO shields with a typical thickness of $\sim 3 \mathrm{~cm}$, which stop protons with energies $\lesssim 100 \mathrm{MeV}$.

The background of irradiated CdTe-DSD was thoroughly investigated with Monte Carlo simulations in our previous work. ${ }^{4}$ In that paper, we successfully reproduced the observed background of CdTe-DSD with the simulations, and revealed that it is dominated by the protoninduced radioactivation of the CdTe-DSD itself.

\section{Monte Carlo Simulations of the HXI Background}

To verify the hypothesis of the origins of the HXI backgrounds in the previous section, we performed a full Monte Carlo simulation by taking into account the in-orbit radiation environment. We used a Monte Carlo simulation framework Comptonsoft, ${ }^{11}$ identical to that used in a previous work on the radioactivation background of the CdTe-DSD. ${ }^{4}$ In the COMPTONSOFT, interactions between incident particles and detector materials are simulated by the software based on the GEANT4 toolkit library. ${ }^{12-14}$ In addition, detailed detector responses due to the charge carrier transport inside semiconductor detectors are also implemented. In this work, we used the same parameter values for the detector response as those tuned to reproduce the experimental data. ${ }^{15}$ Most of the passive materials and the imager module and active shields are implemented in the simulation to accurately calculate interactions in the radiation environment.

\subsection{Radiation Environment}

The radiation environment assumed in this work is shown in Fig. 4. It is composed of albedo neutrons, geomagnetically trapped protons, low-energy electrons, cosmic x-rays, and albedo gamma-rays. The flux and spectral shapes of albedo neutrons, cosmic x-rays, and albedo gamma-rays were based on our previous study. ${ }^{10}$ Geomagnetically trapped protons were obtained from the Space Environment Information System (SPENVIS) ${ }^{16}$ AP-8 model at solar minimum.

The spectral shape of the low-energy electrons was also obtained from the SPENVIS AE-8 model, but its flux was estimated from the observed trigger rate of the top-layer DSSD. Since we eliminated the SAA and the other high flux regions in the analysis (see Hagino et al. ${ }^{15}$ for more details), the electron flux averaged over all the orbital phase obtained from AE- 8 is an overestimation. Although the orbital dependence of the electron flux is implemented in the AE-8 model, the lower flux region included in our analysis is not fully reproduced in the model. Thus, we scale the AE- 8 flux by a ratio of the observed trigger rate in the low flux region to the orbitaveraged trigger rate. The scaling factor is estimated to be $4.2 \times 10^{-4}$ to $6.7 \times 10^{-4}$. In the trapped electron spectrum in Fig. 4, the upper limit of the scaling factor is adopted because

J. Astron. Telesc. Instrum. Syst. $\quad 046003-5 \quad$ Oct-Dec 2020 • Vol. 6(4) 


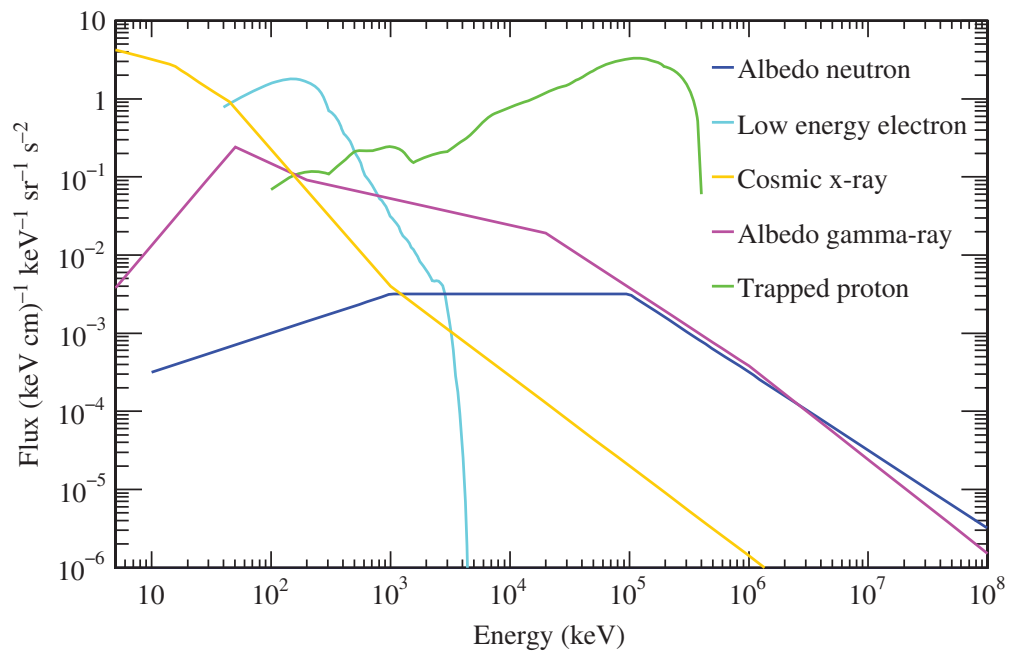

Fig. 4 Radiation environment spectral model of the Monte Carlo simulations.

it gives a simulated background spectrum that matches well with the observed spectrum. It is worth noting that these radiation models except for the low-energy electrons were not optimized for the observed data of the HXI.

\subsection{Comparison with the Observed Spectra}

The simulated background spectrum of each layer is shown in Fig. 5. The observed background spectra are well reproduced by the Monte Carlo simulations with albedo neutrons, low-energy
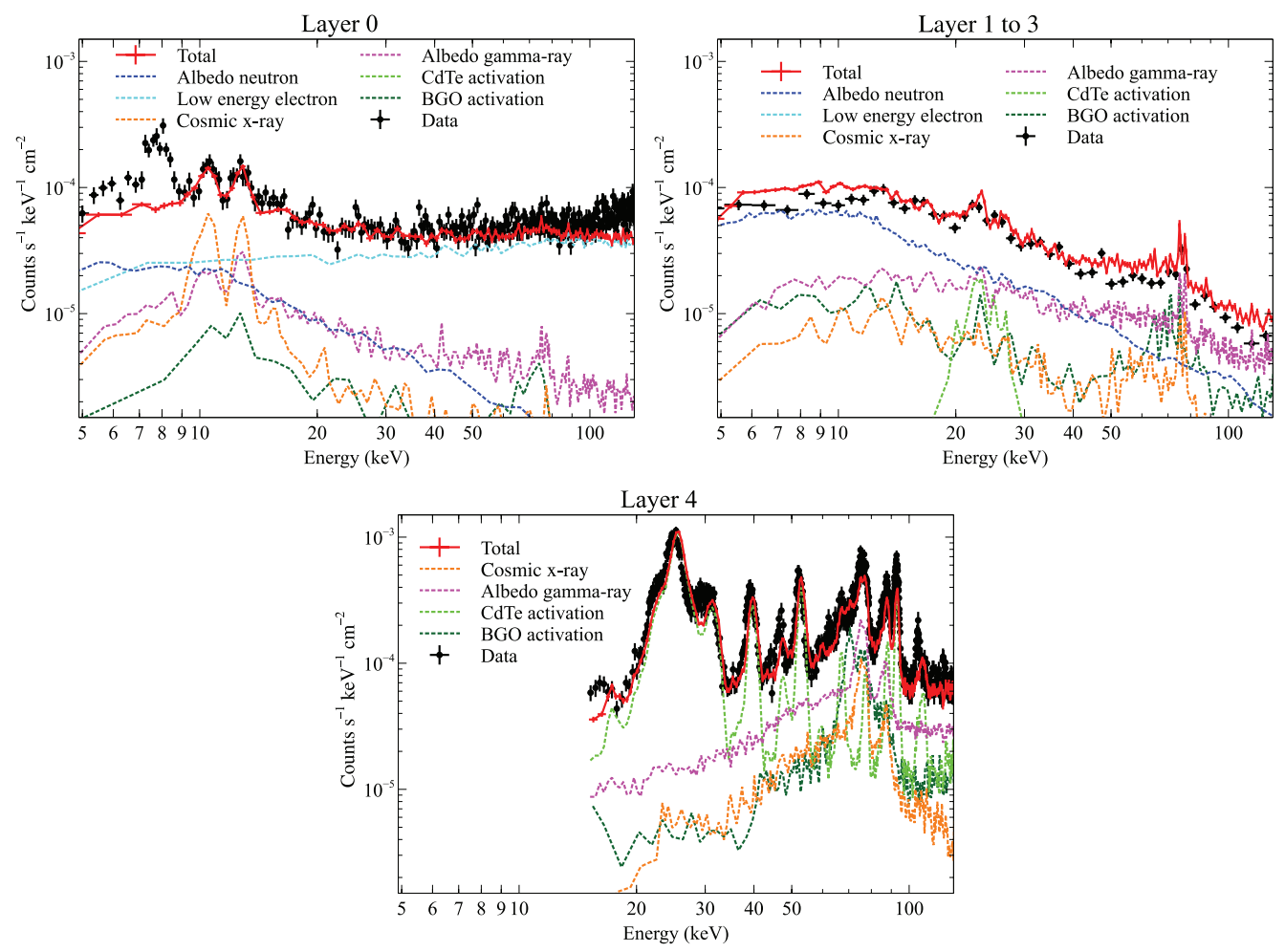

Fig. 5 Simulated background spectra of top-layer DSSD (layer 0), middle-layer DSSDs (layer 1 to 3 ), and CdTe-DSD (layer 4) of the HXI (red solid lines), compared with the observed data (black points). The dashed lines represent the contributions from each component. Albedo neutrons and low-energy electrons are not shown in the bottom panel (layer 4) because their contribution is negligible. 
electrons, trapped protons, cosmic x-rays, albedo gamma rays, and radioactivation of CdTe and BGO. In all the layers, the difference between the simulation results and observations is less than a factor of two, except for the line-like feature at $8 \mathrm{keV}$ in the top-layer DSSD (layer 0). Although the origin of this small excess is unclear, it is possibly due to the fluorescence $\mathrm{x}$-ray of copper at $8 \mathrm{keV}$ induced by the radiation environment in space. These $\mathrm{x}$-rays might be emitted from materials that are not implemented in the simulation, such as the wire harness and/or the extensible optical bench. The other two lines at 10 to $12 \mathrm{keV}$ in the top-layer DSSD are the fluorescence $\mathrm{x}$-rays from the Cosmic X-ray Background (CXB) shield made of lead.

According to the simulations, the background spectra of the top-layer DSSD (layer 0), middlelayer DSSDs (layer 1 to 3), and CdTe-DSD (layer 4) are dominated by low-energy electrons, albedo neutrons, and proton-induced radioactivation, respectively. These results confirm the validity of the hypothesis based on the spectral features of observational data analysis in Sec. 2.

\section{Suppression of Low-Energy Electron Background}

As shown in Fig. 2, the background of the middle-layer DSSDs (layer 1 to 3 ) has been successfully reduced down to $\sim 3 \times 10^{-5}$ counts s $\mathrm{keV}^{-1} \mathrm{~cm}^{-2}$, so that the remaining background is almost only due to the albedo neutron component, which is difficult to reduce. However, the background of the top-layer DSSD (layer 0) is dominated by the electron component, which degrades the sensitivity to below $20 \mathrm{keV}$ where DSSD is most sensitive. Therefore, reducing this electron component is essential to achieve better sensitivity in future missions. In this section, we discuss the background that results from low-energy electrons, and propose a possible design to reduce it.

\subsection{Incident Path of Low-Energy Electrons}

In Secs. 2 and 3, we showed that the low-energy electrons with energies of $\sim 100 \mathrm{keV}$ are the major background component of the top-layer DSSD. However, electrons with energies of $100 \mathrm{keV}$ are easily stopped by $\mathrm{Si}$ with a thickness of $100 \mu \mathrm{m}$. These electrons cannot enter the DSSD directly because the HXI is designed to block all such direct paths. Therefore, to reduce the electron component, it is necessary to understand the incident path of the low-energy electrons.

Figure 6(a) shows the intersections of a vertical plane crossing the detector center $(X=0)$ and the initial path of the low-energy electrons entering the top-layer DSSD. This plot shows the path of the low-energy electrons on the vertical plane $X=0$, assuming that the electrons do not change their direction before reaching this plane. From this figure, we can infer that most of the electrons hit the entrance window (EW; two layers of 30- $\mu \mathrm{m}$-thick poly-carbonate sheets). It
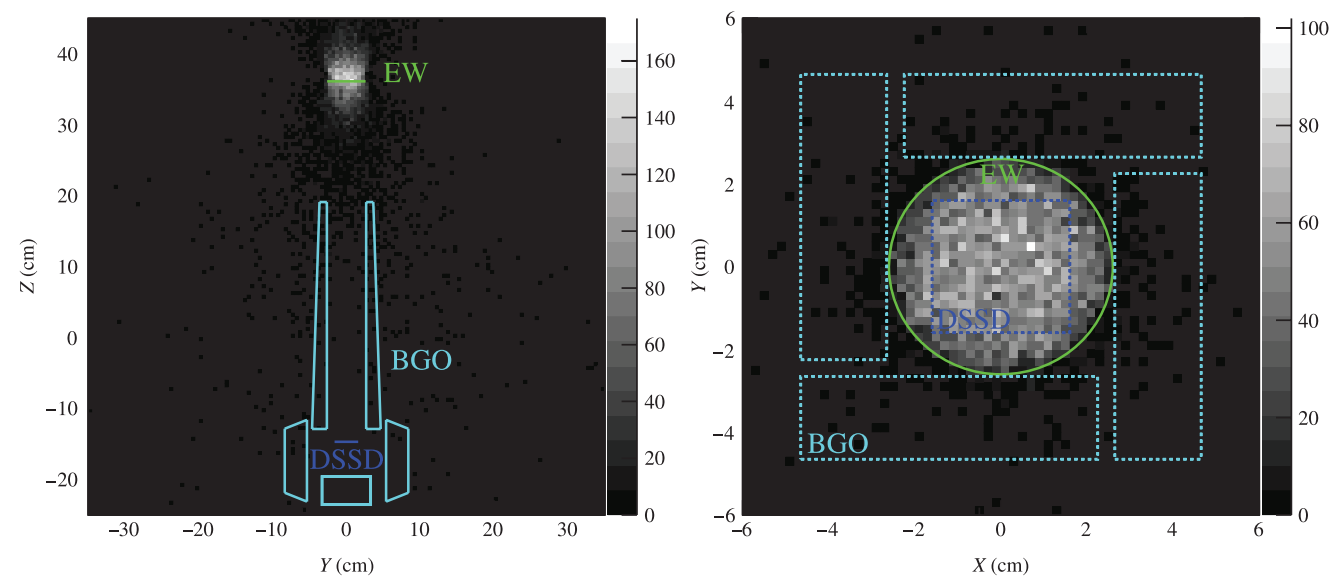

Fig. 6 Incident path of the low-energy electrons. (a) Intersections of the initial electron paths and a vertical plane $(X=0)$. (b) Intersections of the initial electron paths and a horizontal plane at the height of the $\mathrm{EW}(Z=36.1 \mathrm{~cm})$. 
becomes more apparent if we see the intersections of the electron paths with a horizontal plane placed at the height of the EW [Fig. 6(b)]. According to this plot, almost all incident paths intersect with the EW. This indicates that most of the low-energy electrons are scattered at the EW and then enter the top-layer DSSD. Since the EW needs to be thin to avoid absorbing x-rays at $5 \mathrm{keV}$, it is impossible to block the low-energy electrons at the EW. Therefore, to reduce the electron background, it is important to reduce the number of electrons hitting the EW.

\subsection{Cutting Electron Component}

To reduce the low-energy electron background, the EW should be shielded from the low-energy electrons. The shield should have a density thickness more than $\sim 10^{-2} \mathrm{~g} \mathrm{~cm}^{-2}$, corresponding to the continuous slowing down range of $\sim 100-\mathrm{keV}$ electrons. If carbon fiber reinforced polymer (density $\sim 1.5 \mathrm{~g} \mathrm{~cm}^{-3}$ ) is chosen as the electron shield, the required thickness is $<\sim 100 \mu \mathrm{m}$. It is worth mentioning that adding such a shield will not increase the instrumental background since the material from which it is made does not activate. Therefore, this requirement is relatively easy to satisfy.

In addition to the thickness, the electron shield must cover a large solid angle of the sky when seen from the EW. Figure 7 shows the requirement of an opening solid angle of the shield calculated from the simulation data. In this calculation, the shield is assumed to have a circular hole just above the EW. The horizontal axis is the distance between the hole and EW. According to this figure, the solid angle of the hole seen from the EW should be smaller than $0.05,0.01$, and 0.005 sr to reduce the electron background by $1 / 10,1 / 30$, and $1 / 100$, respectively.

As a demonstration, we show the electron background spectrum of the top-layer DSSD with an appropriate shield in Fig. 8. The shield is assumed to cover almost all of the solid angle except for a circular hole with a diameter of $10 \mathrm{~cm}$ at $1 \mathrm{~m}$ above the $\mathrm{EW}$, corresponding to the opening solid angle of $\sim 0.008$. Here, all the electrons coming from the solid angle covered by the shield are assumed to be completely blocked. Since such a shield can efficiently reduce the number of low-energy electrons from outside of the field of view, the electron background spectrum with the shield is nearly two orders of magnitude lower than that without the shield. Hence, as demonstrated, the electron background can be reduced in future missions if an appropriate shielding is applied.

Although we showed that the electron background can be reduced by adding an appropriate shield to the HXI, this design is not necessarily the best one for all hard x-ray missions. The best design must depend on the scientific objectives and constraints on the satellite design. When designing a future mission, a detailed quantitative analysis of the relative merits and demerits of the various possible detector designs would be needed.

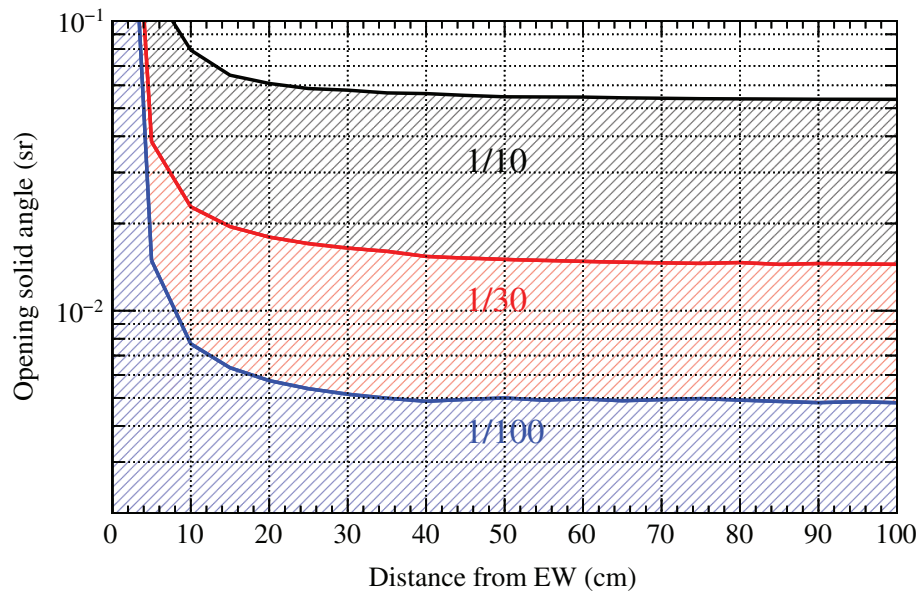

Fig. 7 Opening solid angle of the shield required to reduce the electron background by $1 / 10,1 / 30$, and $1 / 100$. The required solid angle depends on the distance from the EW because the size of the $\mathrm{EW}$ is not negligible at small distances. 


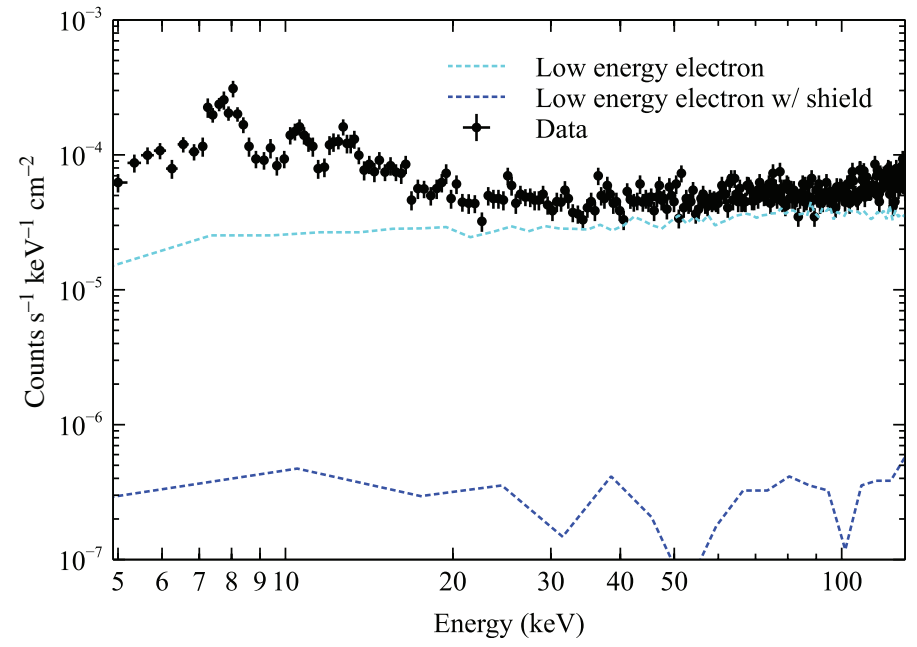

Fig. 8 Simulated electron background spectrum of the top-layer DSSD with shield. With an appropriate shield, the electron background can be reduced. The large fluctuation in the simulated spectrum with shield is due to the Monte Carlo noise.

\section{Conclusions}

The observational data of the HXI indicate the origins of their instrumental backgrounds: the backgrounds of the top-layer Si, middle-layer $\mathrm{Si}$, and bottom-layer CdTe are inferred to originate from low-energy electrons, albedo neutrons, and proton-induced radioactivation, respectively. We successfully reproduced the spectral shape and flux of the observed HXI backgrounds using the full Monte Carlo simulations. Based on the results from this simulation, we conclude that the electron background is expected to be reduced after applying appropriate shielding, which can further benefit future missions.

\section{Acknowledgments}

We acknowledge all the Hitomi team members, including many graduate students, for their great contributions to the HXI and the Hitomi project. We acknowledge the support of Ministry of Education, Culture, Sports, Science, and Technology/Japan Society for the Promotion of Science (JSPS)/ KAKENHI under Grant Nos. 24105007, 15H03639, 25287059, 24244014, and 16H02170, and the JSPS Core-to-Core Program. All the members from the U.S. acknowledge support received from the NASA Science Mission Directorate. Stanford and Stanford Linear Accelerator Center (SLAC) members acknowledge support via Department of Energy contract to SLAC National Accelerator Laboratory DE-AC3-76SF00515 and NASA under Grant No. NNX15AM19G. French members acknowledge support from the Centre National d'Etudes Spatiales.

\section{References}

1. K. Nakazawa et al., "Hard x-ray imager onboard Hitomi (ASTRO-H)," J. Astron. Telesc. Instrum. Syst. 4, 021410 (2018).

2. T. Takahashi et al., "Hitomi (ASTRO-H) x-ray astronomy satellite," J. Astron. Telesc. Instrum. Syst. 4, 021402 (2018).

3. K. Tamura et al., "Supermirror design for hard x-ray telescopes on-board Hitomi (ASTRO-H)," J. Astron. Telesc. Instrum. Syst. 4, 011209 (2018).

4. H. Odaka et al., "Modeling of proton-induced radioactivation background in hard x-ray telescopes: Geant4-based simulation and its demonstration by Hitomi's measurement in a low Earth orbit," Nucl. Instrum. Methods Phys. Res. Sect. A 891, 92-105 (2018).

5. G. Sato et al., "The Si/CdTe semiconductor camera of the ASTRO-H hard X-ray imager (HXI)," Nucl. Instrum. Methods Phys. Res. Sect. A 831, 235-241 (2016). 
6. T. Takahashi et al., "Hard x-ray detector (HXD) on board Suzaku," Publ. Astron. Soc. Jpn. 59, S35-S51 (2007).

7. M. Kokubun et al., "In-orbit performance of the hard x-ray detector on board Suzaku," Publ. Astron. Soc. Jpn. 59, S53-S76 (2007).

8. I. C. Whittaker et al., "Determining the spectra of radiation belt electron losses: fitting DEMETER electron flux observations for typical and storm times," J. Geophys. Res. Space Phys. 118, 7611-7623 (2013).

9. Y. Fukazawa et al., "Modeling and reproducibility of Suzaku HXD PIN/GSO background," Publ. Astron. Soc. Jpn. 61, S17-S33 (2009).

10. T. Mizuno et al., "Monte Carlo simulation study of in-orbit background for the soft gammaray detector on-board ASTRO-H," Proc. SPIE 7732, 77323C (2010).

11. H. Odaka et al., "Development of an integrated response generator for Si/CdTe semiconductor Compton cameras," Nucl. Instrum. Methods Phys. Res. Sect. A 624, 303-309 (2010).

12. S. Agostinelli et al., "Geant4-a simulation toolkit," Nucl. Instrum. Methods Phys. Res. Sect. A 506, 250-303 (2003).

13. J. Allison et al., "Geant4 developments and applications," IEEE Trans. Nucl. Sci. 53, 270-278 (2006).

14. J. Allison et al., "Recent developments in Geant 4," Nucl. Instrum. Methods Phys. Res. Sect. A 835, 186-225 (2016).

15. K. Hagino et al., "In-orbit performance and calibration of the Hard X-ray Imager onboard Hitomi (ASTRO-H)," J. Astron. Telesc. Instrum. Syst. 4, 021409 (2018).

16. "SPENVIS—-space environment, effects, and education system," https://www.spenvis.oma.be.

Kouichi Hagino is an assistant professor at Tokyo University of Science. He received his BS and MS degrees in physics from the University of Tokyo in 2010 and 2012, respectively, and his $\mathrm{PhD}$ in physics from the University of Tokyo in 2015. He has been working in development of semiconductor detectors for applications in high-energy astrophysics.

Biographies of the other authors are not available. 